\title{
Nathen
}

\section{Towards predicting Noise-Power-Distance curves for propeller and rotor powered aircraft}

\author{
Amargianitakis D.C. ${ }^{1}$ \\ Self R.H. ${ }^{2}$ \\ Proença A.R. ${ }^{3}$ \\ Synodinos A.P. ${ }^{4}$, Visiting Academic \\ Institute of Sound and Vibration, University of Southampton \\ Southampton, Hampshire, SO17 1BJ, UK
}

Torija A.J. ${ }^{5}$

Acoustics Research Centre, University of Salford, Manchester M5 4WT, UK

\begin{abstract}
Propeller and rotor based propulsion systems are the predominant choice of power delivery system in the upcoming Urban Air Mobility market. Fully electric air-taxis (car sized vehicles with Vertical Take-off and Landing, VTOL, capabilities) concepts are using the benefits of the scalable properties of electric motors to distribute propulsor units all over the airframe. The large variety of concepts and configurations of these vehicles poses a serious issue in predicting noise generated on the ground. The need for a high-level model to aid in acoustic decision making is evident. Through the demonstrated methodology of computationally deriving Noise - Power - Distance curves for conventional turbo fan aircraft, this paper delivers the capability of dealing with propeller propulsion systems and the associated propeller tonal noise sources to generate the NPDs and therefore noise exposure maps. The aims can be broken down into two objectives: a) demonstrate the capabilities of the proposed propeller harmonics noise scaling laws to calculate noise variation from a baseline scenario and b) incorporate the scaling components into the larger capability of producing noise exposure contours, by the means of computationally deriving Noise-Power-Distance curves for propeller power aircraft. Preliminary NPD curves for General Aviation sized propeller power aircraft are generated and discussed.
\end{abstract}

\footnotetext{
1dca1g14@soton.ac.uk

${ }^{2}$ rhs@soton.ac.uk

${ }^{3}$ A.Proenca@soton.ac.uk

${ }^{4}$ A.Synodinos@soton.ac.uk

${ }^{5}$ A.J.TorijaMartinez@salford.ac.uk
} 


\section{NOMENCLATURE}

\section{SYMBOLS}

$\Omega \quad 2 \pi$ times the shaft rotation frequency

$\Psi_{V}, \Psi_{D}, \Psi_{L}$ normalised source transforms

$\theta \quad$ radiation angle from propeller axis

$B \quad$ number of blades

$B_{D} \quad=b / D$, chord-to-diameter ratio

$C_{D} \quad$ drag coefficient

$C_{L} \quad$ lift coefficient

D propeller diameter

$J_{n} \quad$ Bessel function

$k_{x}, k_{y} \quad$ wave numbers

$m \quad$ harmonic of blade passing frequency

$M_{h}=\sqrt{M_{x}^{2}+M_{t}^{2}}$ helical tip Mach number
$M_{r}=\sqrt{M_{x}^{2}+z^{2} M_{t}^{2}}$ section relative Mach number

$M_{t} \Omega r_{t} / c_{0}$, tip rotational Mach number

$M_{x}=V / c_{0}$, flight Mach number

$n \quad=m B=$ harmonic of shaft frequency

$p \quad$ pressure disturbance

$P_{n} \quad$ complex Fourier coefficient of $p$

$r \quad$ Distance from origin to observer

$r_{t} \quad=D / 2$ propeller tip radius

$t_{b} \quad$ ratio of maximum thickness to chord

$V \quad$ flight speed

$y \quad$ observer distance from propeller axis

$z \quad=r_{0} / r_{t}$ normalised radial coordinate

\section{INTRODUCTION}

The Urban Air Mobility (UAM) market [1] is quickly taking over a major part of research within the aeronautic and aviation industry. While simultaneously investors and financial researchers are evaluating the market growth could create a $\$ 1.5$ trillion market by 2040 [2], with evidence lying in the fact that major airlines placing orders of billions worth of electric vertical take-off and landing (VTOL) aircraft.

Many designers are choosing propeller and rotor propulsion systems alongside fully or hybrid electric energy storage and delivery systems. As an inevitable consequence the study of propeller/rotor noise is regaining the interests of research organisations. The major noise generating mechanisms are basically the same for all rotating blades, whether that be propellers or rotors. These mechanisms can be further broken down into discrete frequency noise (thickness, loading, etc) and broadband components with the relative importance of each depending on design and operating conditions.

In a greater attempt to model the noise of these novel aircraft and their operations in the Institute of Sound and Vibration (ISVR), this paper initiates the development of a framework able to carry out high-level assessment of UAM vehicles early in the design process, when limited input data is available. There is an obvious necessity for tools able to carry out multiple studies at low computational cost to 1 . enable the exploration of a large design space in terms of noise output early in the decision-making process and 2. provide crucial information to manufacturers about the acoustic impact their vehicle will have in the community of operation.

This paper focuses on the purely computational generation of Noise-Power-Distance (NPD) curves of propeller/rotor powered aircraft. NPD curves could then be coupled with traditional airport noise models such as the FAAs AEDT [3] and Eurocontrol's tool IMPACT [4] or high level community noise models such as RANE [5] to calculate noise maps (contours). The framework is based on the knowledge of baseline noise levels of an appropriately chosen baseline aircraft. Then using individual noise source prediction scaling laws for the dominant sources of sound, changes to the sound power 
output, between the baseline and study aircraft are evaluated. Once the initial points of the $L_{A, \max }$ NPDs are calculated, the standard SAE AIR 1845 [6] computational procedure is used to extrapolate to all other NPD distances. SEL NPDs are then also computationally generated by implementing flyovers of a lumped source model comprised of said dominant sources. Finally, to demonstrate the capability of the model, preliminary NPDs for two current propeller aircraft are generated; a single reciprocating engine powered Cessna 172 and the twin engine turboprop de Havilland Canada DHC-6 Twin Otter.

\section{REVIEW OF HANSON FREQUENCY DOMAIN MODEL FOR PROPELLER HARMONIC NOISE}

The development of scaling laws in many cases depends on experimental measurements and data collected for specific sources and operations parameters. These are laws based on empirical or semiempirical models and have proved to be a reliable way of predicting the acoustic field for various aeroacoustics sources; some of these include, fan noise [7], jet noise [8] and airframe noise [9] . In this paper, a simplified version of the frequency domain method developed by Hanson is derived, with the intention of understanding the underlying physics connecting the noise output and key parameters.

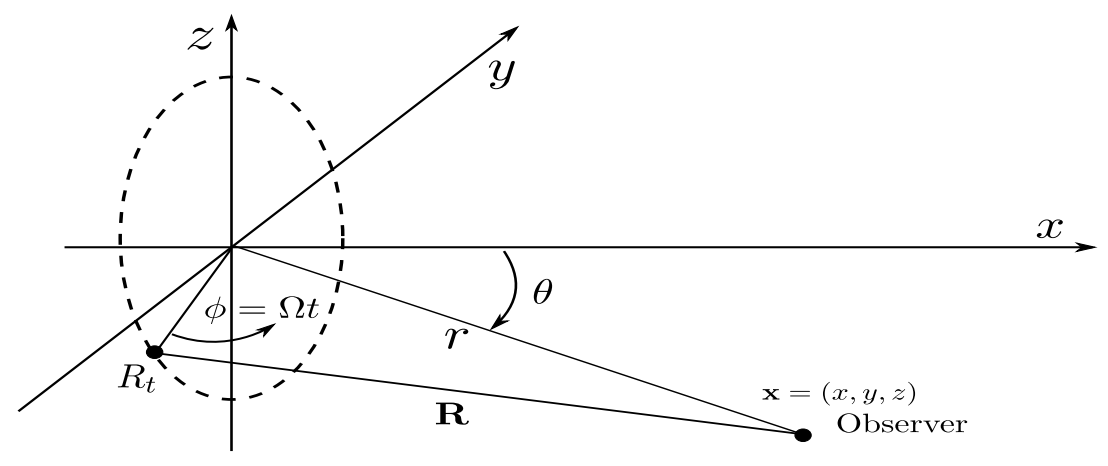

Figure 1: Geometry of rotating source and acoustic field.

As previously mentioned steady loading and thickness noise sources constitute the linear content of the sound field that can be modelled by the linearised equations of motion for an inviscid fluid. The Hanson model is a far-field frequency domain model for a single rotating propeller using a helicoidal surface representation of the blades. Hanson's formulation was chosen in this study as it accounts for the linear thickness and loading sources and the nonlinear quadrupole sources (although emitted due operation regime) including forward flight, and to this day represents a unifying theory of propeller harmonic noise. Hanson published an extension to the method to account for unsteady-loading which will be omitted for the analysis presented within this paper but will be considered in future work.

Hanson's derivation starts at the Goldstein version of the acoustic analogy,

$$
\rho^{\prime}(x, t)=-\frac{1}{c_{0}^{2}} \int_{-T}^{T} \int_{S(\tau)}\left(\rho_{0} V_{n} \frac{\partial G}{\partial \tau}+f_{i} \frac{\partial G}{\partial y_{i}}\right) d \tau+-\frac{1}{c_{0}^{2}} \int_{-T}^{T} \int_{v(\tau)} T_{i j} \frac{\partial^{2} G}{\partial y_{i} \partial y_{j}} d y d \tau
$$

An approximation from thin wing aerodynamic theory is used, permitting the surface boundary conditions to be satisfied on a mean surface rather than on the blade upper and lower surfaces. Thus, the source strengths $V_{n}$ and $f_{i}$ are determined from actual blade geometry but their point of action is on the mean helicoidal surface (To be further discussed in the asymptotic approximation Section 4).

For $B$ blades, the $m^{\text {th }}$ harmonic of blade passing frequency is found by setting $n=m B$ and multiplying by $\Omega$. Waveforms can be computed from the Fourier series. To study the effects of blade design and operation parameters Hanson recast his solution into a form that explicitly displays 
these variables. This form is duplicated here for the ease of the reader and is the starting point of the asymptotic analysis and scaling model within this paper:

$$
\begin{aligned}
\left\{\begin{array}{l}
P_{V m} \\
P_{D m} \\
P_{L m}
\end{array}\right\} & =\frac{\rho_{0} c_{0}^{2} B \sin \beta \exp \left[i m B\left(\frac{\Omega r}{c_{0}}-\frac{\pi}{2}\right)\right]}{8 \pi(y / D)\left(1-M_{x} \cos \beta\right)} \\
& \times \int_{0}^{1} M_{r}^{2} \exp \left(i \phi_{s}\right) J_{m B}\left(\frac{m B z M_{t} \sin \beta}{1-M_{x} \cos \beta}\right)\left\{\begin{array}{c}
k_{x}^{2} t_{b} \Psi_{V}\left(k_{x}\right) \\
i k_{x}\left(C_{D} / 2\right) \Psi_{D}\left(k_{x}\right) \\
i k_{y}\left(C_{L} / 2\right) \Psi_{L}\left(k_{x}\right)
\end{array}\right\} d z
\end{aligned}
$$

with $\phi_{s}$ representing a phase lag due to blade sweep (neglected further on in this study as simple blade geometry is assumed), while $k_{x}$ and $k_{y}$ being dimensionless wave numbers defined by,

$$
\begin{gathered}
k_{x}=\frac{2 m B B_{D} M_{t}}{M_{r}\left(1-M_{x} \cos \theta\right)} \\
k_{y}=\frac{2 m B B_{D}}{z M_{r}}\left(\frac{M_{x}-M_{r}^{2} \cos \theta}{1-M_{x} \cos \theta}\right)
\end{gathered}
$$

In this form, $\Psi_{V}, \Psi_{D}$ and $\Psi_{L}$ represent the Fourier transforms of the three sources giving the thickness and loading distributions of the blade,

$$
\left\{\begin{array}{l}
\Psi_{V}\left(k_{x}\right) \\
\Psi_{D}\left(k_{x}\right) \\
\Psi_{L}\left(k_{x}\right)
\end{array}\right\}=\int_{-\frac{1}{2}}^{\frac{1}{2}}\left\{\begin{array}{l}
H(x) \\
f_{D}(x) \\
f_{L}(x)
\end{array}\right\} \exp \left(i k_{x} x\right) d x
$$

Finally the contributions of the individual sources may be summed to get the Fourier transform coefficient of the pressure at the $m^{\text {th }}$ harmonic of the blade passing frequency $P_{m B}$, while the far-field time domain pressure may be given Fourier transform itself.

\section{SCALING OF PROPELLER HARMONIC NOISE}

The use of analytical formulas for the derivation of scaling laws is advantageous as direct insight to the influence of design geometry and operating conditions have on the fundamental and its harmonics, may be accomplished without full scale experimental procedures. Literature suggests that for propeller harmonic noise not single global scaling law exists (such as Lighthill's eighth power law for jet noise) and rather analytical time or frequency domain models may be used (with the disadvantage a slight increase in time, computational power and inputs requirement) to get much more accurate estimation of the sound power (total acoustic energy) emission [10].

Some of the main assumptions of the Hanson frequency domain model will be transcribed here, as the applicability and limitation range of the approximations and scaling laws will directly correlate to starting model. The problem is treated assuming propeller in flight with uniform flow. The source terms used in the derivation are, the monopole (volume), dipole (loading) and quadrupole (Lighthill's stress tensor neglecting viscosity), however the quadrupole term is ignored as it becomes significant at transonic blade section speeds [11] (general aviation applications generally consider a maximum blade tip mach number of 0.8 or $M_{t}<0.8$ ). The "thin-blade" approximation permits the mathematical simplification of replacing the blade surfaces by their effect on the fluid, this results in errors only at very high harmonic order, and out of the plane of rotation. Finally, Doppler factors in both amplitude 
and frequency roles are contained with the model, although the relatively low cruise speeds of general aviation vehicles in question are not strongly affected.

The Bessel function makes an appearance in all harmonic noise terms $P_{V m}, P_{D m}$ and $P_{L m}$, it largely contributes to the spectral shape of the radiated noise and the overall level and is sometimes referred to a "radiation-factor". The argument $m B z M_{t} \sin \beta /\left(1-M_{x} \cos \beta\right)$ takes maximum values within the plane off rotation, that is $\beta=90^{\circ}$, and is equal to $m B z M_{t}$. For tip Mach numbers applicable to general aviation vehicles the argument $m B z M_{t}$ remains approximately equal (or slightly smaller) to the order $m B$ of the Bessel functions where its value peaks. This behaviour may be approximated using the small argument asymptotic formula. For the ease we introduce the variable $\alpha_{1}$ where $\alpha_{1}=$ $\sin \beta /\left(1-M_{x} \cos \beta\right)$, therefore the asymptotic approximation of the Bessel function in Equation 2 may be given by,

$$
J_{m B}\left(m B M_{t} \alpha_{1}\right) \sim \frac{1}{(m B) !}\left(\frac{m B M_{t} \alpha_{1}}{2}\right)^{m B}
$$

The source terms (the terms in braces of the integral in Equation 2) may be broken down into term in two components: a Spectrum shape component and a spectrum level component. The $\Psi$ terms represent the effect of chordwise noncompactness, that is, interference at the observer location of signals emitted from various source locations along the chord, contributing to the spectrum shape definition. Chordwise blade geometry and loading is what determines these characteristics, therefore we assume a simple parabolic thickness distribution and uniform lift and drag distribution. The thickness, drag, and lift noise components are directly proportional to the thickness ratio $t_{b}$, drag coefficient $C_{D}$, and lift coefficient $C_{L}$, respectively. Combining the noncompactness effect relation along with the proportionality we may estimate the source term transforms by,

$$
\begin{gathered}
\Psi_{V}\left(k_{x}\right) \approx \frac{1}{k_{x}^{2}}=\left(\frac{1-M_{x} \sin \theta}{2 m B B_{D} M_{t}}\right)^{2} \\
\Psi_{D}\left(k_{x}\right)=\Psi_{L}\left(k_{x}\right) \approx \frac{1}{k_{x}}=\left(\frac{1-M_{x} \sin \theta}{2 m B B_{D} M_{t}}\right)
\end{gathered}
$$

Therefore the individual source contributions may be estimated as simple functions of the blade thickness, $t_{b}$ and blade loading, $C_{L}$ and $C_{D}$,

$$
\begin{gathered}
k_{x}^{2} t_{b} \Psi_{V}\left(k_{x}\right) \approx t_{b} \\
k_{x}\left(C_{D} / 2\right) \Psi_{D}\left(k_{x}\right) \approx C_{D} / 2 \\
k_{x}\left(C_{L} / 2\right) \Psi_{L}\left(k_{x}\right) \approx \frac{C_{L} k_{y}}{2 k_{x}} \approx \frac{\left(M_{x}-M_{h}^{2} \cos \theta\right)}{M_{t}} \frac{C_{L}}{2}
\end{gathered}
$$

The dominating term within the integral in the radial direction is the square of the section relative Mach number, $M_{r}^{2}$. The section relative Mach number take maximum at the tip of the propeller, and is equal to the helical Mach number $M_{h}$. We may estimate the value of the integral by assuming it is directly proportional to $M_{h}^{2}$, and for a static case proportional to $M_{t}^{2}$, as can be seen,

$$
M_{r}^{2} \approx M_{h}^{2}=M_{x}^{2}+M_{t}^{2}
$$

This assumption implies that the thickness and loading sources are applied on the propeller tip section. To correct the thickness and loading parameters to account for the entire blade we assume the thickness ratio at the blade tip takes the average value over the blade, $t_{b}(z=1)=\overline{t_{b}}$, and the loading coefficient follow accordingly, $C_{D}(z=1)=\bar{C}_{D}$ and $C_{L}(z=1)=\bar{C}_{L}$ (Note: the loading parameters 
$C_{D}$ and $C_{L}$ may instead be defined in terms of thrust and torque, $d T / d z$ and $d Q / d z$ respectively, with the integral over the radius giving the thrust and torque acting on the blade).

Combining of the above mentioned procedures and adding the contributions of the individual sources together we may estimate $P_{m B}=P_{V m}+P_{D m}+P_{L m}$ as,

$$
\begin{aligned}
P_{m B} & \approx\left(\frac{\rho_{0} c_{0}^{2}}{8 \pi}\right)\left(\frac{D}{y}\right)\left(\frac{\sin \theta}{\left(1-M_{x} \cos \theta\right)}\right) M_{h}^{2}\left(\frac{1}{(m B)}\right)\left(\frac{m B M_{t} \sin \theta}{2\left(1-M_{x} \cos \theta\right)}\right)^{m B} \\
& \times\left[\bar{t}_{b}+\frac{\bar{C}_{D}}{2}+\frac{\bar{C}_{L}\left(M_{x}-M_{h}^{2} \cos \beta\right)}{2 M_{t}}\right] \\
& \times \exp \left[i m B\left(\frac{\Omega r}{c_{0}}-\frac{\pi}{2}\right)\right]
\end{aligned}
$$

As we are interested in the sound power output as a function of design and operational parameter we must first estimate the intensity which is proportional to $p_{r m s}^{2}$, therefore we have,

$$
\begin{aligned}
p_{m, r m s}^{2} & =\left\langle P_{m B} P_{m B}^{*}\right\rangle \approx \underbrace{\left(\frac{\rho_{0} c_{0}^{2}}{8 \pi}\right)^{2}}_{\text {constants }} \underbrace{\left(\frac{1}{y}\right)^{2}}_{\text {Spherical Spreading }} \underbrace{D^{2} M_{h}^{4}\left[\overline{t_{b}}+\frac{\bar{C}_{D}}{2}+\frac{\bar{C}_{L}\left(M_{x}-M_{h}^{2} \cos \beta\right)}{2 M_{t}}\right]^{2}}_{\text {Design/operation parameters }} \\
& \times \underbrace{\left(\frac{\sin \theta}{\left(1-M_{x} \cos \theta\right)}\right)^{4 m B+2}}_{\text {Directivity }} \underbrace{\left(\frac{1}{(m B) !}\right)^{2}\left(\frac{m B M_{t}}{2}\right)^{2 m B}}_{\text {Spectral Shape }}
\end{aligned}
$$

The asymptotic approximation allows for some simple observations to be made regarding the influence of the tip Mach number. Assuming as previously, $M_{t}<1$ the $M_{t}^{2 m B}$ term in Equation 14 shows that at a given rotation rate propellers with larger blade number generate less sound and furthermore the contribution of the higher harmonics rapidly decreases with increasing harmonic order $m$ while the tip rotation speed remains subsonic. This allows for a modelling technique used by Heidmann for the estimation of fan discrete-tone and combination-tone noise [7], to be applied. The procedure involves predicting the spectrum level and spectrum shape. The spectrum level is defined at each design/operation point by the fundamental tone, while the levels of remaining spectral frequencies are estimated by referencing the blade passing frequency and the spectral shape function. However, unlike Heidmann the spectrum shape is not constant, rather a function of the blade number and tip mach number.

The $\sin \theta$ present within the Bessel function argument, and therefore in the approximation relation, causes highly documented dipole behaviour of the propeller. The radiated noise diminishes towards the propeller axis as approached from both front and rear directions. Additionally, the Doppler factor $1-M_{x} \cos \beta$ ) causes a shift of that directivity pattern into the forward hemisphere with respect to the propeller plane.

The overall sound power output of the system may therefore be evaluated through the average intensity emitted over angles $\beta$ and $\varphi$, which may be evaluated in the far field through the root-meansquare,

$$
W=4 \pi R^{2} \bar{I}=\frac{4 \pi R^{2}}{\rho_{0} c_{0}} \int_{0}^{2 \pi} \int_{0}^{\pi} p_{r m s}^{2} R^{2} \sin \beta d \beta d \varphi
$$


The calculation of $\bar{I}$ pressure depends on the integration over the spherical surface surrounding the source. The integral over $\varphi$ takes a trivial answer as rotational symmetry exists around the propeller axis. When considering the evaluation of the integral over the polar angle $\beta$ over in the interval $\beta \in(0, \pi)$ the solution is no longer trivial. The problem may be divided into two cases, a. a static (or hovering) propeller, meaning $M_{x}=0$ and $\mathrm{b}$. the case where mean flow is non-zero, $M_{x} \neq 0$ and assuming subsonic flight speeds $\left(M_{x}<1\right)$.

The evaluation for the two cases are omitted for terseness, and will included in future publications. In addition the methodology following in Section 5 allows for calculation of the variation of sound power emitted including directivity effects, therefore the overall sound energy quantification bypassed.

\section{NOISE VARIATION DUE TO CHANGES}

To incorporate the effect of changing engine power settings, the derived equations are functions of propeller and aircraft operational parameters (thrust setting, torque, rpm e.t.c.) that vary between operational procedures.

It has been previously demonstrated that the use of changes in noise, or $\triangle \mathrm{PWL}$, as compared to a baseline scenario gives more accurate prediction than attempting to evaluate absolute values directly using the scaling laws. Coupled with the capability of using $\triangle \mathrm{PWL}$ in order to generate Noise-Power-Distance curves the use of the scaling formulation may be extended to computationally evaluating modified rotor designs in an urban airport environment through the use of exposure metrics and contours.

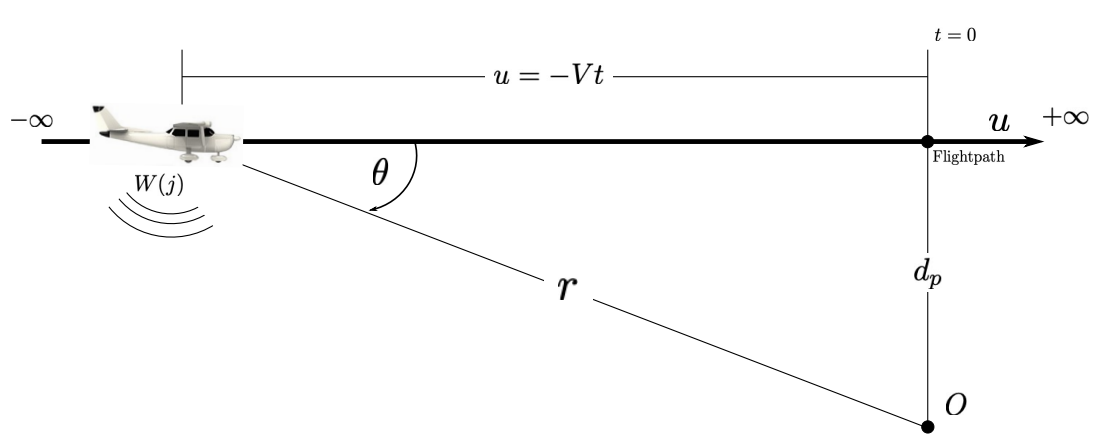

Figure 2: Diagram of typical flyover procedure for obtaining NPD data.

The basis of this methodology assumes that any noise level (PWL) of the subject ( modified) aircraft is given in terms of the sum of a baseline aircraft noise level and the difference in noise level between the two aircraft,

Considering a baseline aircraft, it consists of noise sources emitting overall sound power $W_{0}$. As a result of operational or technological changes, the acoustic noise sources on the aircraft may change in number, magnitude and other acoustic properties, e.g. spectral content, therefore denoting $n$ the total number of noise sources after the modifications and $s$ an individual noise source that changed by acoustic power $\Delta W_{s}$ we may write,

$$
W=W_{0}+\sum_{s=1}^{n} \Delta W_{s}
$$

noting that for noise sources that previously did not exist, $\Delta W_{s}=W_{s}$ and for sources that were removed, $\Delta W_{s}=-W_{s}$. Therefore in order to calculate the acoustic emission of the novel aircraft design, knowledge on the acoustic output of the baseline aircraft is required, along with the individual changes of the acoustic sources. As mentioned, Synodinos [12] demonstrated this methodology for jet, fan and airframe noise sources on conventional turbofan aircraft, and applied it on novel 
ducted fan configurations (considering the same acoustic sources [13]). Following we will extend the methodology to incorporate the propeller harmonic noise sources though the use of scaling expressions developed in the previous sections. The methodology includes an elegant way of including the tonal spectral content of propeller noise when calculating the change in noise $(\Delta$ Noise) between configurations. This is achieved through the use of a spectral shape function. In this paper, a spectral shape function is explored through the use of the scaling expressions and therefore a product of the starting Hanson model, however other functions may be used to define the spectral content shape according to the application.

The remaining part of this section is dedicated to deriving the change in noise (or specifically the change in SPL) emitted by a propeller aircraft, considering loading and thickness noise sources. The propeller discrete tone noise (as it pertains to the mentioned sources) of any aircraft may be expressed as the energy sum across all harmonics. The individual harmonic levels may be related to the fundamental tone by some spectra shape function, allowing for the SPL to be written as,

$$
L_{p, 0}=10 \log _{10}\left(\sum_{m=1}^{\infty} 10^{\left[L_{f_{1}, 0}+F_{1}\left(\frac{f}{f_{1}, 0}\right)\right] / 10}\right)
$$

with $f=m B \Omega$ and $f_{1}=B \Omega$ represent the frequency of mode $m$ and the fundamental tone respectively, while $L_{f_{1}, 0}$ is the SPL of the baseline fundamental. $F_{1}$ denotes the spectra shape function.

When modifications in terms of operational and/or design parameters are made, changes to the fundamental tone, $\Delta L_{f_{1}}$ and the shape of the harmonics $\Delta F_{1}$ are observed, leaving the new SPL as,

$$
L_{p}=10 \log _{10}\left(\sum_{m=1}^{\infty} 10^{\left[L_{f_{1}, 0}+F_{1}\left(\frac{f}{f_{1}, 0}\right)+\Delta L_{f_{1}}+\Delta F_{1}\left(\frac{f}{f_{1}}\right)\right] / 10}\right)
$$

where $L_{f_{1}}=L_{f_{1}, 0}+\Delta L_{f_{1}}$ represents the new level of the fundamental tone, and $L_{m}(f)=L_{f_{1}}+$ $\Delta F_{1}\left(f / f_{1}\right)$ represents the new sound level of the harmonics (narrowband spectrum).

Returning to Equation 14, we may consider a case where modifications to a baseline aircraft are made. The change in level of the fundamental frequency observed may be estimated by setting $m=1$ in both cases and taking the difference between the two, leaving,

$$
\Delta L_{f_{1}}=10 \log _{10}\left(\frac{p_{f_{1}}^{2}}{p_{f_{1}, 0}^{2}}\right)
$$

or

$$
\begin{aligned}
\Delta L_{f_{1}} & =20 \log _{10}\left(\frac{y}{y_{0}}\right)+20 \log _{10}\left(\frac{B D}{B_{0} D_{0}}\right)+40 \log _{10}\left(\frac{M_{h}}{M_{h_{0}}}\right) \\
& +20 \log _{10}\left(\frac{t_{b}+C_{D} / 2+C_{L}\left(M_{x}-M_{h}^{2} \cos \beta\right) /\left(2 M_{t}\right)}{t_{b, 0}+C_{D, 0} / 2+C_{L, 0}\left(M_{x, 0}-M_{h, 0}^{2} \cos \beta_{0}\right) /\left(2 M_{t, 0}\right)}\right) \\
& +20 \log _{10}\left(\frac{B_{0} !}{B !}\right)+20 \log _{10}\left(\frac{\left(B M_{t}\right)^{B}}{\left.B_{0} M_{t, 0}\right)^{B_{0}}}\right)+20 \log _{10}\left(\frac{\left[\sin \beta /\left(1-M_{x} \cos \beta\right)\right]^{2 B+1}}{\left[\sin \beta_{0} /\left(1-M_{x, 0} \cos \beta_{0}\right)\right]^{2 B_{0}+1}}\right)
\end{aligned}
$$

where the subscript 0 denotes the values corresponding to the condition before the modifications were applied (baseline).

Separating all terms including the mode number $m$ in Equation 14 we may define a function $S$. This function determines the shape of the harmonics.

$$
S(m)=\left(\frac{\sin \beta}{1-M_{x} \cos \beta}\right)^{4 m B+2}\left(\frac{1}{(m B) !}\right)^{2}\left(\frac{m B M_{t}}{2}\right)^{2 m B}
$$


Normalising this expression by its value for $m=1$,

$$
\frac{f}{f_{1}}(m)=\frac{S(m)}{S(1)}=\left(\frac{\sin \beta}{1-M_{x} \cos \beta}\right)^{4 B(m-1)}\left(\frac{B !}{(m B) !}\right)^{2}\left(\frac{B M_{t}}{2}\right)^{2 B(m-1)} m^{2 m B}
$$

noting that $\frac{f}{f_{1}}(1)=1$ by definition. Finally the change in level of the harmonics $(m>1)$ between a baseline case and a modified counterpart may be given by,

$$
\begin{aligned}
\Delta F_{1}\left(\frac{f}{f_{1}}\right) & =20 m \log _{10}\left(\frac{m^{B}}{m^{B_{0}}}\right)+20 \log _{10}\left(\frac{B !\left(m B_{0}\right) !}{B_{0} !(m B) !}\right) \\
& +20 \log _{10}\left(\frac{\left(B M_{t}\right)^{B(m-1)}}{\left(B_{0} M_{t, 0}\right)^{B_{0}(m-1)}}\right)+40 \log _{10}\left(\frac{\left[\sin \beta /\left(1-M_{x} \cos \beta\right)\right]^{B(m-1)}}{\left[\sin \beta_{0} /\left(1-M_{x, 0} \cos \beta_{0}\right)\right]^{B_{0}(m-1)}}\right)
\end{aligned}
$$

\section{NOISE-POWER-DISTANCE CURVE GENERATION}

This section presents the methodology of computationally generating NPDs using the knowledge of how propeller noise changes as a function of changes in the design and operational parameters. The same approach to the derivation of computational NPDs can be seen in [12], where NPDs for fan, jet and airframe noise sources were calculated. The work in this section is novel with regards to the propeller noise sources, and aims on extending the methodology presented in said publications.

The steps in producing NPD curves for propeller powered aircraft from a baseline measured NPD point and aircraft noise variation due to design or operational changes are almost identical to that of conventional aircraft, although differences do occur in the treatment of the spectral content of the sound levels, as changes to the discrete tonal content are also accounted for by the propeller scaling model. For brevity reasons a detailed explanation is omitted but is intended for future publications. For detailed explanation the reader is encouraged to follow the procedure described in [12] and [14] along with the SAE AIR1845 [6] computational step.

\section{PRELIMINARY NPDS FOR PROPELLER POWERED AIRCRAFT}

In order to validate while at the same time demonstrating the capability of the scaling laws in conjunction with the $\triangle \mathrm{PWL}$ methodology, this section estimates both $L_{A, \max }$ and SEL NPD curves of two different propeller powered aircraft and compares to published data [15].

For each aircraft a baseline point is chosen (depicted with a red cross in Figures 3 and 4, this point corresponds to a specific power setting $j$ and slant distance $d$ ) on the $L_{A, \max }$ curves, according to the type of operation of interest. Using the single baseline value and the methodology described in Sections 4, 5 and 6 predictions for the rest of the NPD slant distances and power setting are made. The first validation case is the Cessna 172 (Maximum take-off weight (MTOW) 1,114 kg). The chosen NPD point corresponds to that of slant distance equals $304.8 \mathrm{~m}$ (or 1,000 ft). Figure 3 show the departure NPDs for the Cessna 172 at two specific power setting; 59.6\% and 100\% of maximum static thrust (MST) respectively. The published data may be seen in dashed lines, while the predicted in solid ones.

The second validation case demonstrates the capability of estimating NPD for slightly larger turboprop (MTOW 5,682 kg, still within the "small" noise category of aircraft defined by the ICAO Annex 16 [16]) with a twin engine configuration. Figure 4 compares the published and predicted take-off NPD curves (using same notation) at this time $30 \%$ and $100 \%$ of MST. 


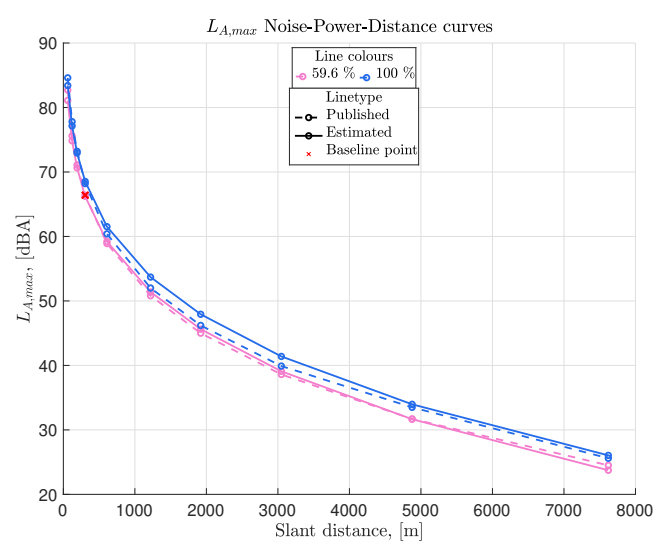

(a) $L_{A, \max }$

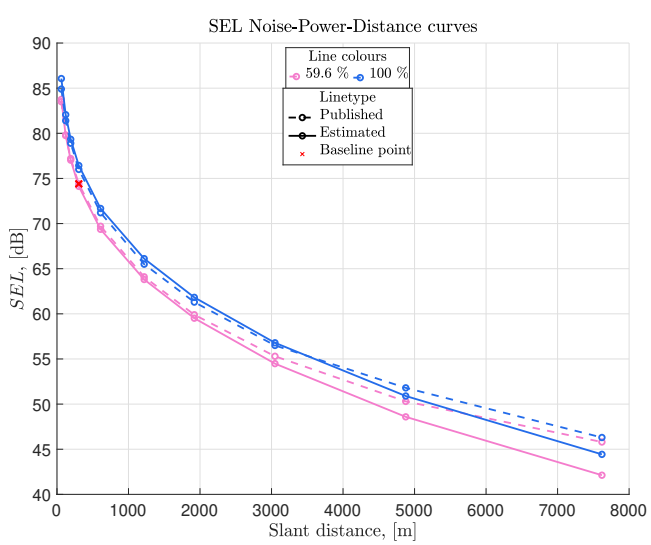

(b) SEL

Figure 3: Published and predicted departure NPDs for a single engine Cessna 172 Skyhawk.

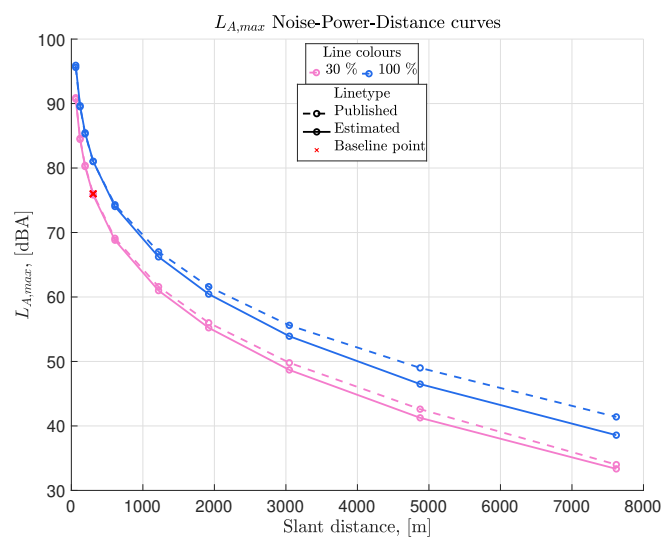

(a) $L_{A, \max }$

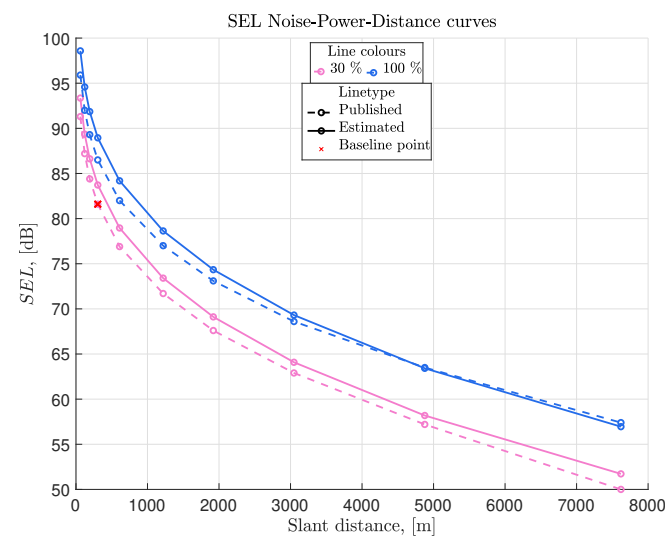

(b) SEL

Figure 4: Published and predicted departure NPDs for twin engine de Havilland Canada DHC-6 (Twin Otter)

\section{CONCLUSIONS}

The work presented within this paper summarises the methodology of computing NPD curves for propeller/rotor power aircraft. A scaling law of the dependence of the fundamental tone of the harmonic noise due to thickness and loading source is coupled with a spectral shape function to fully define the discrete tonal content of a propeller source. Using a reference baseline, the change in overall noise output due to changes in propeller parameters is calculated. This change in noise is then used to computationally derive Noise-Power-Distance curves, which serve as the main input for noise exposure map models.

The validation / case studies presented within this paper represent initial results that are tied to a few significant simplification assumptions. Namely a) both types of NPD curves presented ( $L_{A, \max }$ and SEL) are strongly dependant to the overall directivity of the aircraft. In this initial study the assumption of a simple dipole source directivity was made. This assumption is closely related to the next assumption discussed, that of a single dominant acoustic noise source. The aircraft is represented by the assumed dominant propeller tonal noise. Propeller tonal noise due to loading and thickness source as discussed by Hanson in [17], [18], [19] and others [20], [21]. Realistic directivity data of propeller aircraft flyovers could potentially reduce the error in both types of NPD curves. b) the propeller and specifically propeller tonal noise is assumed to be the dominant source. As of 
this preliminary study this assumption results in the negligence of other possible aeroacoustic noise sources (e.g. airframe noise, reciprocating engine noise, jet noise etc. ) that may be present. This assumption may be justified for takeoff procedures as high thrust requirements lead to heavily loaded propeller blades contributing to the discrete loading noise. However, approach procedures may suffer from the opposite effect of low loading characteristics. Therefore future approach calculations will consider addition model to account for airframe noise etc, as in [12]. In the case of the Cessna 172, the reciprocating engine noise is assumed to be effectively masked by the propeller noise [22]. The same hold for turboprop engine architectures as in the case of the DHC-6, where the combustion and jet noise sources are considered negligible with respect to the propeller source [23]. Finally, c) the final point of discussion brings two points into one. The frequency content of the predicted NPD curves depends on two factors, 1 . the baseline spectra and 2. the predicted changes to the spectra by the scaling model. Baseline spectral data (specifically spectral shapes) for different operational regimes are not readily available. The predictions within this paper are based on spectral shapes of flyover measurements at the highest possible power setting (100\% MST). The predicted changes to the spectra may also lead to errors as discrete-tonal noise of the propeller is only accounted for (e.g. changes in high-frequency vortex broadband noise are not captured). As mentioned closely coupled with the frequency content of the sound in the atmospheric attenuation. As the attenuation is related to the propagation distance and the frequency of the sound, the large NPD slant distances tend to amplify the error associated with the prediction of the spectra shape change.

Nevertheless, despite the sources of error discussed, the framework provided estimates with an average error of $\pm 1.5 \mathrm{~dB}$ for the $L_{A, \max }$ NPD curves and $\pm 2 \mathrm{~dB}$ for the SEL NPD curves, which is within the tolerance suggested by the ECAC Doc 29 [14]. Finally, it worth noting that the framework is by nature computational therefore eliminating errors associated with experimental measurements of flyover procedures.

It may therefore be concluded that the framework has the potential to provide good NPD estimates for propeller aircraft, as mentioned, when limited data is available at the early stages of design. Future publications will aim at demonstrating the application of the framework on novel fully electric fixed wing and rotorcraft designs and their and contemporary operations.

\section{REFERENCES}

[1] R. Goyal. Urban air mobility (uam) market study. Technical report, National Aeronautics and Space Administration (NASA), November 2018.

[2] Morgan Stanley Research. Are flying cars preparing for take-off? https://www . morganstanley . com/ideas/autonomous-aircraft, January 2019.

[3] J. Koopmann, A. Hansen, S. Hwang, M. Ahearn, and G. Solman. Aviation environmental design tool (aedt) version 2c technical manual. Technical Report Tech. Rep. DOT-VNTSC-FAA-16-17, Federal Aviation Administration (FAA), July 2016.

[4] EUROCONTROL. Integrated aircraft noise and emissions modelling platform. https://www.eurocontrol.int/platform/ integrated-aircraft-noise-and-emissions-modelling-platform.

[5] A. J. Torija, R. H. Self, and I. H. Flindell. A model for the rapid assessment of the impact of aviation noise near airports. The Journal of the Acoustical Society of America, (2):981-995, 2017.

[6] Society of automotive engineers: Procedure for the calculation of aircraft noise in the vicinity of airports. Technical Report 1845, SAE AIR, 1981.

[7] M. Heidmann. Interim prediction method for fan and compressor source noise. 1975.

[8] James R. Stone, Donald E. Groesbeck, and Charles L. Zola. Conventional profile coaxial jet noise prediction. AIAA Journal, 21(3):336-342, 1983. 
[9] Martin R. Fink. Noise component method for airframe noise. AIAA paper, October 1977.

[10] Siyang Zhong, Peng Zhou, Ryu Fattah, and Xin Zhang. A revisit of the tonal noise of small rotors. Proceedings of the Royal Society A: Mathematical, Physical and Engineering Sciences, 476(2244):20200491, 2020.

[11] D.B. Hanson and M.R. Fink. The importance of quadrupole sources in prediction of transonic tip speed propeller noise. Journal of Sound and Vibration, 62(1):19-38, 1979.

[12] A. P. Synodinos, R. H. Self, and A. J. Torija. A framework for predicting noise-power-distance curves for novel aircraft designs. Journal of Aircraft, 2017.

[13] Athanasios Synodinos, Rod Self, and Antonio Torija. Noise assessment of aircraft with distributed electric propulsion using a new noise estimation framework. 072017.

[14] Report on standard method of computing noise contours around civil airports, vol. 2: Technical guide. Tech. rep. ecac.ceac doc. 29, 4th ed, European Civil Aviation Conference (ECAC), December 2005.

[15] Eurocontrol Experimental Centre. Aircraft noise and performance (anp) database v2.1, 2016.

[16] ICAO. Annex 16 to the convention on international civil aviation: Environmental protection, volume i aircraft noise. Technical report, International Civil Aviation Organization, July 2017.

[17] D. B. Hanson and B. Donald. Helicoidal surface theory for harmonic noise of propellers in the far field. AIAA J., 18(10):1213-1219, October 1980.

[18] D. B. Hanson and B. Donald. Influence of propeller design parameters on far field harmonic noise in forward flight. AIAA J., 18(11):1313-1319, 1980.

[19] D. B. Hanson and B. Donald. Near-field frequency-domain theory for propeller noise. AIAA J., 23(4):499-504, April 1985.

[20] B. Magliozzi. Noise characteristics of a model counterrotating prop-fan. AIAA J., 1987.

[21] W. K. Blake. Mechanics of Flow-Induced Sound and Vibration, volume 2. Elsevier Inc., 2017.

[22] H. H. Hubbard. Aeroacoustics of flight vehicles: Theory and practice:volume 1: Noise sources. Technical report, NASA Langley Research Center, Hampton, Virginia, 1991.

[23] F. Farassat, S.L. Padula, and M.H. Dunn. Advanced turboprop noise prediction based on recent theoretical results. Journal of Sound and Vibration, 119(1):53-79, 1987. 